\title{
Trigger level track reconstruction in CMS with a fully time-multiplexed architecture using a Hough transform implemented in an FPGA
}

\section{Luigi Calligaris* $\dagger$}

STFC Rutherford Appleton Laboratory, Didcot, UK

E-mail: luigi.calligarisestfc.ac.uk

\begin{abstract}
A new tracking system is under development for operation in the CMS experiment at the High Luminosity LHC. It includes an outer tracker which will construct "stubs" - pairs of hits built by correlating clusters measured in two closely spaced silicon sensor layers - and transmit them offdetector at $40 \mathrm{MHz}$. If tracker data is to contribute to keeping the Level-1 trigger rate at around $750 \mathrm{kHz}$ under increased luminosity, a crucial component of the upgrade will be the ability to identify tracks with transverse momentum above $3 \mathrm{GeV} / \mathrm{c}$ by building tracks out of stubs. A concept for an FPGA-based track finder using a fully time-multiplexed architecture is presented, where track candidates are identified using a projective binning algorithm based on the Hough Transform. A hardware system based on the MP7 MicroTCA processing card has been assembled, demonstrating a realistic slice of the track finder in order to help gauge the performance and requirements for a full system. This document outlines the system architecture and algorithms employed, highlighting some of the first results from the hardware demonstrator and discusses the prospects and performance of the completed track finder.
\end{abstract}

38th International Conference on High Energy Physics

3-10 August 2016

Chicago, USA

\footnotetext{
* Speaker.

$\dagger$ For the CMS collaborators and the Time Multiplex Track Trigger group. Supported by EU-FP7-PEOPLE-2012ITN project nr. 317446, INFIERI "Intelligent Fast Interconnected and Efficient Devices for Frontier Exploration in Research and Industry".
} 


\section{Upgrading the CMS experiment for the High Luminosity LHC}

The CMS collaboration operates its general-purpose detector [1] at the Large Hadron Collider [2], searching for new physics at the energy frontier and improving the understanding of the the Standard Model of Particles. Increasing the luminosity of the LHC machine is beneficial to its physics program, extending the reach of future particle searches and improving the precision in the measurement of rare Standard Model processes, such as the couplings of the SM Higgs boson.

The High Luminosity LHC (HL-LHC) [3] is an important upgrade program for the collider, planned to be completed by year 2026, after which the instantaneous luminosity will be increased from the current values of $1 \cdot 10^{34}$ to $5 \cdot 10^{34} \mathrm{~cm}^{-2} \mathrm{~s}^{-1}$ and, if the machine allows it, to ultimate values of $7 \cdot 10^{34} \mathrm{~cm}^{-2} \mathrm{~s}^{-1}$. The latter values correspond, respectively, to a number of 140 and 200 concurrent proton-proton collisions per bunch crossing (pile-up, or PU), and represent conditions significantly more challenging than those the current detectors were designed for. A program for the upgrade of the CMS detector [4] is ongoing, to enable it to cope with the increased luminosity while keeping the desired level of performance. This upgrade is referred to as the Phase-2 Upgrade.

\section{The Phase-2 Upgrade of the CMS Outer Tracker}

One of the main issues related to the increased number of pile-up interactions is the degradation in performance of the hardware trigger (Level-1 trigger), since it is based uniquely on calorimeter and muon chamber information. The solution proposed for the CMS Phase- 2 upgrade [4] involves including silicon tracker information at this level of the trigger. The upgraded outer tracker is engineered for read out at the full collision rate of $40 \mathrm{MHz}$ of all pairs of hits, further referred to as stubs, compatible with tracks with a $p_{T}>2-3 \mathrm{GeV} / c^{2}$. This latter cutoff reduces the required bandwidth, and is implemented in a configuration known as $p_{T}$-module $[5,6]$ where closely-spaced pairs of detectors are read-out by a dedicated ASIC that correlates the hits detected in them. A schematic of the logic is shown in Fig. 1.

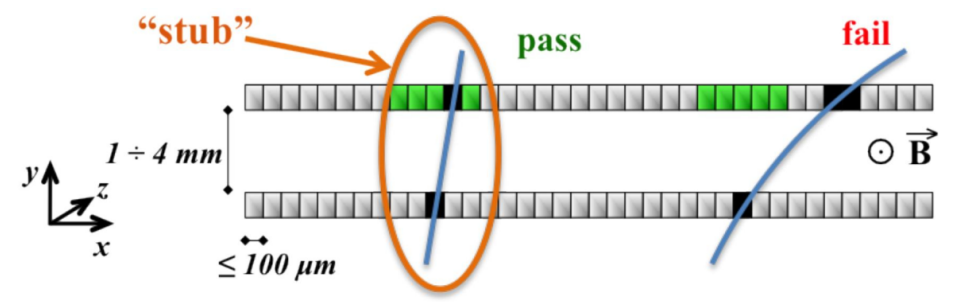

Figure 1: An illustration of the logic that selects pairs of hits (black squares) compatible with a track with a $p_{T}$ higher than a set threshold: if a hit in the inner detector has a corresponding hit measured in the compatible region (green squares), the two hits are paired to form a stub.

\section{Particle track reconstruction with a Hough Transform}

In the proposed implementation [7], stubs from an event are read out from the tracker modules and collected by data, trigger and control (DTC) cards, which perform preliminary calculations, such as the conversion from sensor local coordinates to detector global coordinates in the CMS reference system [1], and deliver the stubs from a specific event and corresponding to one octant 
in azimuth of the outer tracker to their assigned track processor. As the time in which a track reconstructor cannot accept new stubs is longer than the interval between events, a time multiplexing scheme is employed, distributing events to identical instances of the reconstruction system.

Tracks are found by means of a Hough Transform (HT) [8], which is a method to test a set of data points for mutual compatibility with respect to a pre-established relationship, which in this case is the alignment of stubs along the projection of the helical trajectory on the $r-\phi$ plane. This is a circular trajectory since the magnetic field in CMS is normal to the latter plane. For particles with a sufficiently high momentum, the small arc of circular trajectory described inside the detector can be approximated with an Archimedean spiral, with equation $\phi=\phi_{0}+a r$. With a choice of the variables reflecting the measurements in the tracker, the equation becomes

$$
\phi_{T}=\phi_{\text {stub }}+k \cdot\left(q / p_{T}\right) \cdot\left(r_{\text {stub }}-T\right) \equiv \phi_{\text {stub }}+k \cdot\left(q / p_{T}\right) \cdot r_{T}
$$

where $T$ is a fixed reference radius (currently a value of $58 \mathrm{~cm}$ is used, half-way across the tracker inner and outer radii), $\phi_{T}$ is the azimuth of the trajectory at this radius, $\phi_{\text {stub }}$ is the azimuth of the stub, $r_{T}$ is the difference between the radius of the stub and the reference radius, $\mathrm{k}$ is a constant proportional to the magnetic field and $\left(q / p_{T}\right)$ is the ratio between charge and transverse momentum of the particle. For each stub (Fig. 2a) with values $\left(\phi_{\text {stub }}, r_{T}\right)$ assigned to a HT track finder, the value of $\phi_{T}$ as a function of $\left(q / p_{T}\right)$ is calculated. In the space of the transformed variables $\left(q / p_{T}\right)$ and $\phi_{T}$, stubs appear as straight lines (Fig. 2b) and cross at the values that correspond to those of the track. To find tracks, a histogram (Fig. 2c) counts the number of stubs in different tracker layers that cross its cells in Hough space, counting only stubs that cross the $p_{T}$-module with an inclination compatible with the cell $\left(q / p_{T}\right)$ hypothesis. Cells with a count above a threshold (in this case 4 or 5 , configurable to suit geometry and detector conditions) identify a candidate track.

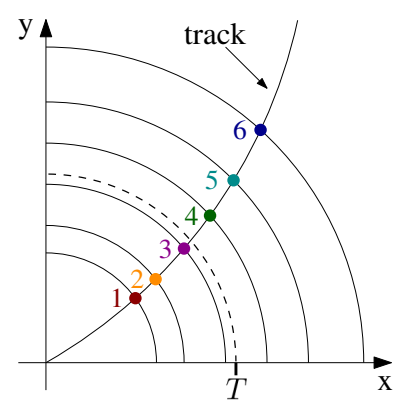

(a) View of a track in the $r-\phi$ plane and its associated stubs.

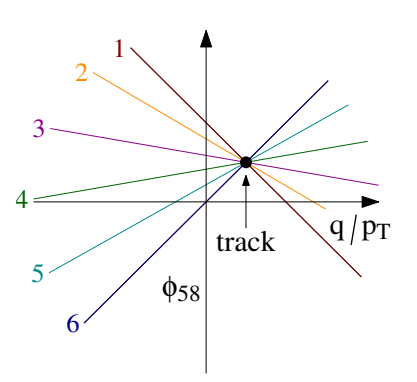

(b) The same stubs as lines in (c) Track finding histogram in

Hough-transformed space. Hough transform space.

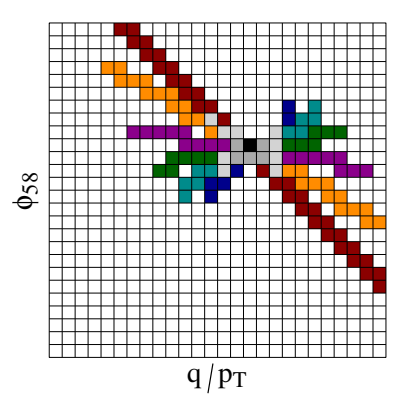

Figure 2: Illustration of the procedure used in track finding with a Hough transform.

To optimise bandwidth, latency and resource utilisation, a number of HT track finders run in parallel, with each of them responsible for a small segment in $\phi-\eta$ space. In the current system, 36 segments run in parallel following a $18(\eta) \times 2(\phi)$ segmentation of the tracker octant. The distribution of stubs to the correct track finder is operated by a dedicated router, called Geometrical Processor (GP). Once track candidates are found in the $r-\phi$ plane, they are filtered by downstream processors that check the consistency of their constituent stubs with a track in the $r-z$ plane $(R Z$ filter) and eliminate duplicate track candidates that are built from the same stubs, as a result of aliasing in the HT histogram binning (duplicate removal). A final stage fits the track trajectories. 


\section{Implementation in FPGA}

The track reconstruction system is being demonstrated [7] by implementing its constituent processors in Imperial Master Processor 7 cards [9], based on a Xilinx Virtex-7 XC7VX690T FPGA, and daisy-chaining them via the 72 input and 72 output 10-Gbps optical links on-board each card.

Many different implementations of the HT track finder have been investigated, and the one that showed the best results has the stubs propagating in the HT histogram along a daisy chain of columns of cells in $\left(q / p_{T}\right)$, where each of the columns performs independently and in parallel the $\phi_{T}$ calculation and the stub counting. The recently introduced improvement of allowing for a flexible threshold of 5 or 4 stubs in the histogram improves tracking efficiency in the barrel/tracker transition region, as shown in Fig. 3a. Improvements were able to reduce the number of fake tracks, with average occupancies per histogram column of around 6\% (Fig. 3b).

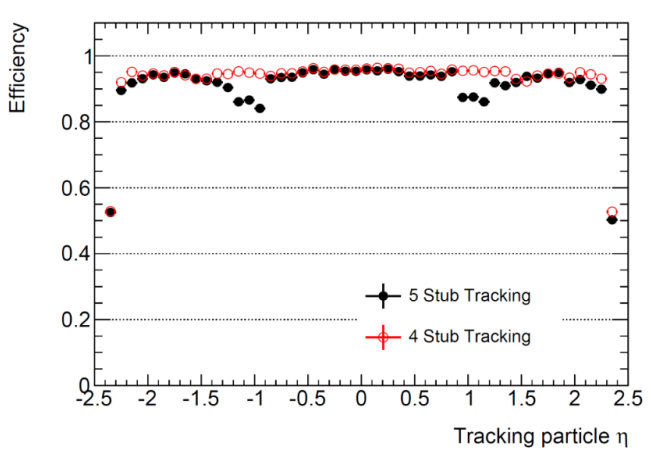

(a) Tracking efficiency as a function of $\eta$.

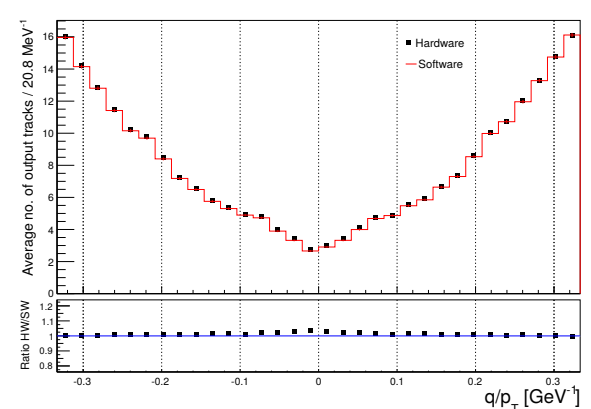

(b) Average number of track candidates for each HT histogram column in $\left(q / p_{T}\right)$.

Figure 3: Results from simulation (Fig. 3a) and hardware tests (Fig. 3b) of the HT track finder.

\section{Conclusions and outlook}

A time multiplexed track finder for the Phase-2 Level-1 trigger upgrade of the CMS detector is being demonstrated in hardware, and its existing components are constantly being improved with promising results. Meanwhile, the implementation of the other filtering and track fitting processors is currently being finalised, these remaining components are being integrated in the full track reconstruction system and the performance of the latter will be measured soon.

\section{References}

[1] S. Chatrchyan et al. [CMS Collaboration], JINST 3 (2008) S08004. doi:10.1088/1748-0221/3/08/S08004

[2] L. Evans and P. Bryant, JINST 3 (2008) S08001. doi:10.1088/1748-0221/3/08/S08001

[3] G. Apollinari, I. Béjar Alonso, O. Brüning, M. Lamont and L. Rossi. doi:10.5170/CERN-2015-005

[4] D. Contardo, M. Klute, J. Mans, L. Silvestris, J. Butler, CERN-LHCC-2015-010

[5] J. Jones, G. Hall, C. Foudas, A. Rose, CERN-2005-011 130-134. doi:10.5170/CERN-2005-011

[6] M. Pesaresi, CERN-THESIS-2010-083. 
[7] C. Amstutz et al., 2016 IEEE-NPSS Real Time Conference (RT). doi:10.1109/RTC.2016.7543102

[8] P. V. C. Hough, US Patent 3,069,654

[9] K. Compton et al., JINST 7 (2012) C12024. doi:10.1088/1748-0221/7/12/C12024 